OPEN ACCESS

Edited by:

Wei Huang,

Chinese Academy of Sciences, China

Reviewed by:

Nabil l. Elsheery,

Tanta University, Egypt

Weimin Ma,

Shanghai Normal University, China

*Correspondence:

Hualing M

mih/@sippe.ac.cn

Specialty section: This article was submitted to

Plant Physiology,

a section of the journal

Frontiers in Plant Science

Received: 05 August 2015

Accepted: 20 January 2016

Published: 15 February 2016

Citation:

Xu M, LV J, Fu P and Mi H (2016)

Oscillation Kinetics of

Post-illumination Increase in Chl

Fluorescence in Cyanobacterium

Synechocystis PCC 6803.

Front. Plant Sci. 7:108.

doi: $10.3389 /$ fpls.2016.00108

\section{Oscillation Kinetics of Post-illumination Increase in Chl Fluorescence in Cyanobacterium Synechocystis PCC 6803}

\author{
Min $X u^{1}$, Jing $L v^{2}$, Pengcheng $F u^{2}$ and Hualing $M i^{1 *}$ \\ ${ }^{1}$ National Key Laboratory of Plant Molecular Genetics, Institute of Plant Physiology and Ecology, Shanghai Institutes for \\ Biological Sciences, Chines Academy of Sciences, Shanghai, China, ${ }^{2}$ Renewable Energy Research Center, China University \\ of Petroleum, Beijing, China
}

After termination of longer-illumination (more than $30 \mathrm{~s}$ ), the wild type of Synechocystis PCC 6803 showed the oscillation kinetics of post-illumination increase in Chl fluorescence: a fast phase followed by one or two slow phases. Unlike the wild type, ndh-B defective mutant M55 did not show any post-illumination increase under the same conditions, indicating that not only the fast phase, but also the slow phases were related to the $\mathrm{NDH}$-mediated cyclic electron flow around photosystem I (PS I) to plastoquinone (PQ). The fast phase was stimulated by dark incubation or in the presence of Calvin cycle inhibitor, iodoacetamide $(\mathrm{IA})$ or cyclic photophosphorylation cofactor, phenazine methosulphate (PMS), implying the redox changes of $P Q$ by electrons generated at PS I reduced side, probably $\mathrm{NAD}(\mathrm{P}) \mathrm{H}$ or ferredoxin ( $\mathrm{Fd})$. In contrast, the slow phases disappeared after dark starvation or in the presence of IA or PMS, and reappeared by longer re-illumination, suggesting that they are related to the redox changes of $P Q$ by the electrons from the photoreductants produced in carbon assimilation process. Both the fast phase and slow phases were stimulated at high temperature and the slow phase was promoted by response to high concentration of $\mathrm{NaCl}$. The mutant M55 without both phases could not survive under the stressed conditions.

Keywords: post-illumination increase in Chl fluorescence, Synechocystis sp. PCC 6803, NAD(P)H dehydrogenase, cyclic electron flow around photosystem I, plastoquinone

\section{INTRODUCTION}

Similar to eukaryotic phototrophs, cyanobacteria carry out oxygen photosynthesis with two distinguishable photosystems, photosystem I (PS I) and photosystem II (PSII; Scherer, 1990). Light energy is converted into biochemical energy by driven transfer of electron from water to terminal electron acceptor $\mathrm{NADP}^{+}$. Thus, the linear electron flow produces ATP by a proton gradient across the thylakoid membrane, coupling the formation of NADPH. In addition to the linear electron flow, there is a cyclic electron flow around PS I, which converts light to electrochemical potential for synthesis of ATP. Cyclic electron flow around PS I is considered physiological important not only for its providing extra ATP for carbon assimilation (Schurman et al., 1972; Mills et al., 1978; Slovacek et al., 1978), but also for its adjusting the production ratio of ATP to NADPH for developmental stages (Hatch, 1987), environmental stress (Mi et al., 2001; Wang et al., 2006), and 
physiological requirements ( $\mathrm{Xu}$ et al., 2014). Since lacking organelles such as chloroplasts and mitochondria, respiratory electron transport chain of cyanobacteria couples with photosynthetic intersystem chain by sharing some components (Jones and Myers, 1963). The respiratory electron transport which can produce ATP in darkness and remove oxygen plays an important role in nitrogen fixation (Scherer et al., 1988).

In cyanobacteria, respiration and cyclic electron flow are mediated by cyanobacterial type- 1 NAD(P)H dehydrogenase (NDH-1) complexes which functions in a variety of bioenergetic reactions (Mi et al., 1992) and $\mathrm{CO}_{2}$ uptake (Ogawa, 1991). NDH1 consists of 17 subunits at least, among those, NdhA-NdhK are homologous to those of Escherichia coli complex I (Friedrich and Scheide, 2000), NdhL-NdhQ specially exist in cyanobacteria, identified by functional proteomics approach (Prommeenate et al., 2004; Battchikova et al., 2005) or by purification (Nowaczyk et al., 2011). NDH-1 from cyanobacteria is speculated to possess an oxygenic photosynthesis-specific (OPS) domain (Birungi et al., 2010) comprised of NdhL-NdhO identified in Synechocystis 6803 (Prommeenate et al., 2004; Battchikova et al., 2005). Several $\mathrm{NDH}$ subunits function in stabilization of NDH-1. NdhQ is also essential for stabilization of the large complex of NDH1 (Zhao et al., 2015). NdhP is involved in the respiratory and cyclic electron flow (Schwarz et al., 2013) and is essential to stabilize the NDH-1L complex (Zhang et al., 2014). NdhS as a novel subunit of NDH-1 participates in the cyclic electron flow in Arabidopsis (Yamamoto et al., 2011) and also in cyanobacteria (Battchikova et al., 2012) that it serves as the docking site for $\mathrm{Fd}$, accepting electrons from Fd in chloroplasts (Yamamoto and Shikanai, 2013).

Post-illumination increase in Chl fluorescence has been found in C4 plant (Asada et al., 1993), C3 plant (Mano et al., 1995), and cyanobacteria (Mi et al., 1995). The phenomenon was explained as the reduction of plastoquinone (PQ) by the electrons from photoreductants accumulated in the stroma or cytosol during illumination, and may reflect cyclic electron transport around PS I mediated by NDH-1 (Mi et al., 1995) and by plastid NADH dehydrogenase-like complex (NDH; Sazanov et al., 1998; Shikanai et al., 1998). It could be used as a convenient way to study the activity of cyclic electron flow around PS I in vivo.

In this work, we found that after termination of illumination, the wild type of Synechocystis sp. PCC 6803 (hereafter Synechocystis PCC 6803) showed the oscillation kinetics of postillumination increase in Chl fluorescence. The oscillation kinetics was further investigated under different conditions combined with the ndhB defective mutant (M55) and inhibitors. The results showed that the oscillation is suggested to be related to the changes in the redox state of PQ by electrons from various photoreductants and responses to environmental stresses.

\section{MATERIALS AND METHODS}

\section{Culture of Cyanobacteria}

Synechocystis PCC 6803 and its $n d h \mathrm{~B}$ defective mutant, M55 (Ogawa, 1991), were cultured in the BG-11 medium buffered with Tris- $\mathrm{HCl}\left(5 \mathrm{mM}, \mathrm{pH} 8.0\right.$; Allen, 1968) at $27^{\circ} \mathrm{C}$ under fluorescent lamps at $60 \mu \mathrm{mol} \mathrm{m} \mathrm{m}^{-2} \mathrm{~s}^{-1}$ bubbling with $2 \% \quad \mathrm{CO}_{2}$ in air. According to the experiments, the cells at logarithmic growth stage were used for measurements.

\section{Measurement of the Kinetics of Changes in Chl Fluorescence}

The redox change of PQ was monitored by Chl fluorescence, using a PAM Chlorophyll Fluorometer (Walz, Effeltrich, Germany) with an emitter-detector-cuvetter assembly (ED-101 US). Details for fluorometer setup were described previously (Schreiber et al., 1994, 1995). The cell suspension was pipetted in a cuvetter with a thermostat. Cells were exposed to the actinic light $\left(\mathrm{AL}, 3 \mathrm{Wm}^{-2}\right)$ for $30 \mathrm{~s}$. Then $\mathrm{AL}$ was turned off and the transient increase kinetics in chlorophyll fluorescence was detected. Each sample was adapted in the dark for $2 \mathrm{~min}$ prior to measurement. As for treatment with the cyclic photophosphorylation cofactor, $100 \mu \mathrm{M}$ of phenazine methosulphate (PMS) was added to the sample before measurement; and with the Calvin cycle inhibitor, iodoacetamide (IA) was pre-incubated with the sample at low concentration $(40 \mu \mathrm{M})$ and high concentration $(1 \mathrm{mM})$. As for dark starvation, cells of Synechocystis PCC 6803 were pre-treated in dark for 24$32 \mathrm{~h}$ before measurement. Every experiment was repeated three times at least.

\section{Oxygen Exchange}

The evolution of oxygen under the white light $\left(150 \mathrm{~W} \mathrm{~m}^{-2}\right)$ was determined in a reaction mixture that contained the midlogarithmic cell of Synechocystis PCC 6803 in the cultural BG11 medium at $5 \mu \mathrm{g} \mathrm{Chl} \mathrm{ml}^{-1}$ with $10 \mathrm{mM} \mathrm{NaHCO}_{3}$ with a Clarktype oxygen electron. The reaction mixture kept at indicated temperatures as shown in Figure $\mathbf{3 A}$ with a thermostat.

\section{RESULTS}

\section{Oscillation Kinetics of Post-illumination Increase in ChI Fluorescence}

The post-illumination increase in Chl fluorescence is used to reflect the activity of cyclic electron flow around PS I in cyanobacteria and high plants. The kinetics usually is monophasic under certain conditions in the previous studies (Deng et al., 2003). Here we found that after termination of the longer-illumination(more than $30 \mathrm{~s}$ ) of red light $\left(3 \mathrm{~W} \mathrm{~m}^{-2}\right)$, the wild type of Synechocystis PCC 6803 showed a oscillation of post-illumination increase in Chl fluorescence: a fast phase, peak1 (P1) followed by one or two slow phases peak2 (P2) or peak3 (P3) (Figure 1). In contrast, $n d h \mathrm{~B}$ defective mutant M55 did not show any post-illumination increase under the same conditions (Figure 1), indicating that not only fast phase, but also slow phases related to the reduction of PQ by the $\mathrm{NDH}$-mediated cyclic electron flow. To know what kinds of components contribute to the different phases, we checked effect of a cyclic electron cofactor and a Calvin cycle inhibitor as follows. 


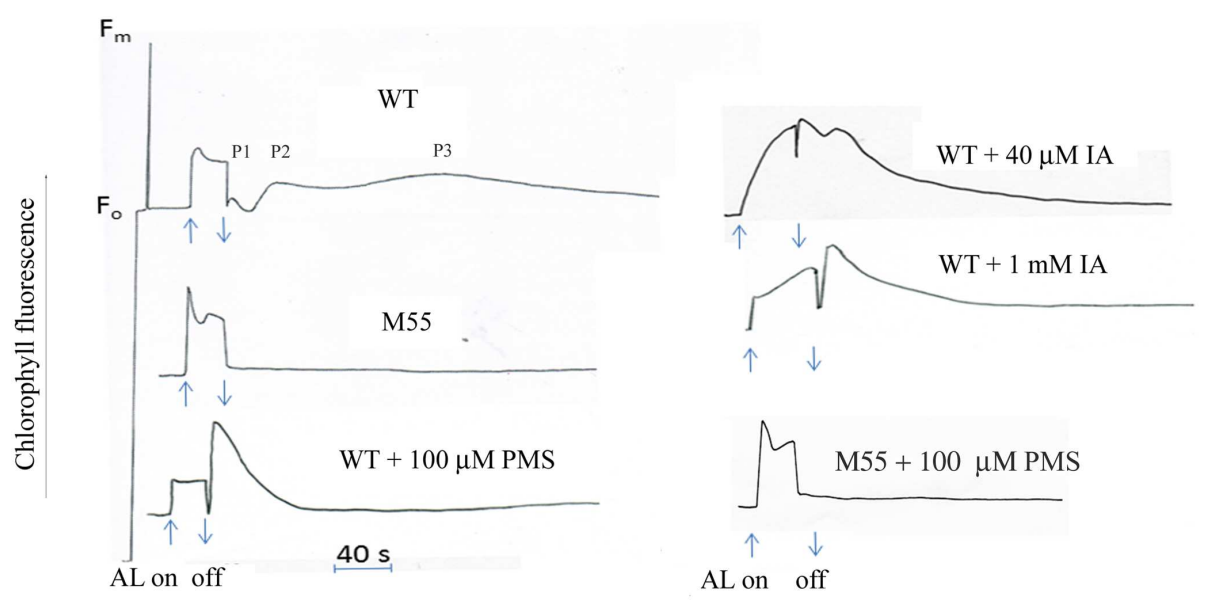

FIGURE 1 | Kinetics of post-illumination increase in Chl fluorescence. The Top curve shows a typical trace of chlorophyll fluorescence in wild type (WT) Synechocystis PCC 6803. The cell at mid-logarithmic stage (OD730 $\approx 0.5)$ was pipetted in a cuvetter with a thermostat. After the cells were exposed to the AL $\left(3 \mathrm{Wm}^{-2}\right)$ for $30 \mathrm{~s}$, the transient increase kinetics in chlorophyll fluorescence was detected. The wild type showed the oscillation kinetics of post-illumination increase in Chl fluorescence: a fast phase, peak1 (P1) followed by one or two slow phase peak2 (P2) or peak3 (P3). M55, NdhB defective mutant; PMS, phenazine methosulphate; IA, iodoacetamide. Each sample was adapted in the dark for 2 min prior to the measurement. The chemicals were, respectively, added to the sample before adaptation in the dark. Every experiment was independently repeated three times at least.

\section{Effect of Inhibitors on the Kinetics of Post-illumination Increase in Chl Fluorescence}

Phenazine methosulphate is a well-known artificial cyclic photophosphorylation cofactor, which bypasses electrons from PS I acceptor side to the P700 (Trebst, 1974). Addition of PMS significantly stimulated the increase of fast phase, but suppressed the slow phases of post-illumination increase in Chl fluorescence in the wild type, but not in M55 (Figure 1), suggesting that the fast phase is caused by the electron donation from simple substrates, probably NADPH, and slow phase is caused by electron donation from the substrates produced in the process of carbon assimilation, and NDH-1 is involved in these processes. To test the possibility, the effect of IA, an inhibitor of Calvin cycle which blocks linear electron flow but stimulates cyclic electron flow around PS I in spinach chloroplasts (Joliot and Alric, 2013), on post-illumination increase in Chl fluorescence was checked. Both the P1 and P2 were significantly stimulated but the P3 disappeared at the presence of low concentration of IA ( $40 \mu \mathrm{M}$; Figure 1). Moreover, high concentration of IA (1 mM) stimulated the fast phase but suppressed the slow phase (Figure 1). The results indicate that fast phase reflects the redox change of PQ by electrons from PS I reduced side, probably NADPH or Fd and the slow phases by those from complicate components synthesized through the Calvin cycle.

\section{Effect of Dark Starvation and Re-illumination on the Oscillation Kinetics of Post-illumination Increase in Chl Fluorescence}

Given that the redox state of PQ is also affected by donation of electrons from respiratory substrates, the effect of dark incubation on the oscillation kinetics was carried out. It has been known that dark starvation for 24-32 h could cause a shortage of respiratory substrates, but the cells still retained their photosynthetic capacity in the Synechocystis cells (Mi et al., 1994). With dark incubation, P1 increased, while P2 and P3 decreased (Figures 2A,B). P1 reached to the maximal value after $24 \mathrm{~h}$, P3 and P2 disappeared after 4.5 and $15 \mathrm{~h}$ dark incubation, respectively (Figures 2B,C). When the 25-h dark-starved cells were re-illuminated for $20 \mathrm{~min}$, the level of P1 fell close to that before the dark incubation while $\mathrm{P} 2$ and $\mathrm{P} 3$ rose to the level before dark incubation (Figures 2D,E). These results suggest that the electrons from PS I reduced side contributes to P1 and respiratory substrates such as glucose and other intermediate such as triose phosphate might affect the P2 and P3.

\section{Effect of Temperature on Kinetics of Post-illumination Increase in Chl Fluorescence}

To further understand the relation of redox changes of PQ with photosynthetic activity, the response of the oscillation kinetics to different temperatures was measured. As shown in Figure 3A, the peak height of both the fast and slow phases and rate of oxygen evolution increased with the rise of temperature and reached to the maximal values until $25-35^{\circ} \mathrm{C}$, respectively. The three parameters decreased dramatically when the temperature reached $45^{\circ} \mathrm{C}$. These results indicate that both the fast phase and slow phase of post-illumination increase in Chl fluorescence display a similar temperature response with the photosynthetic electron transport and other reactions consuming energy, which are all enhanced with the rise of temperature within the limits of physiological temperature. To know the physiological significance of the kinetics, the growth of wild type and NdhB defective mutant M55 without both kineticses has been compared 


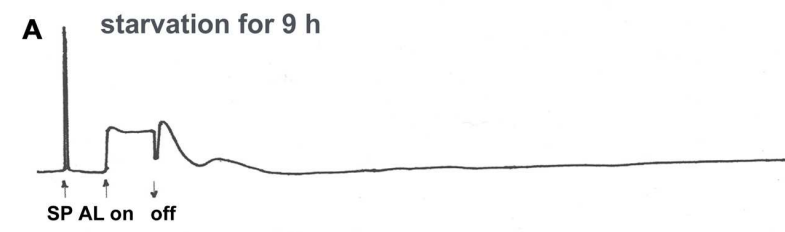

C

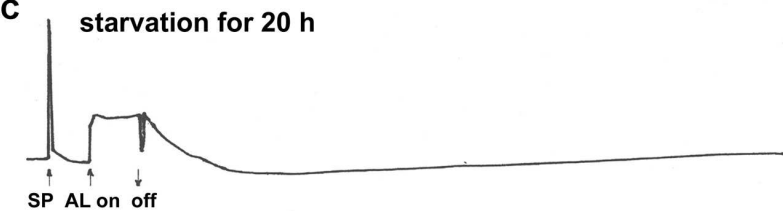

D

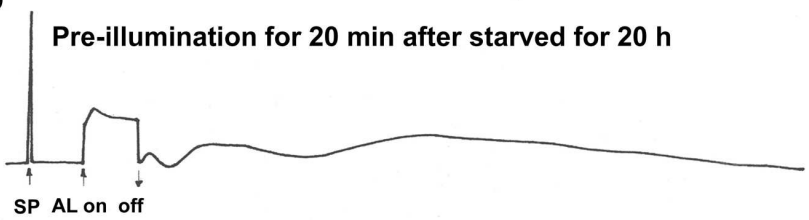

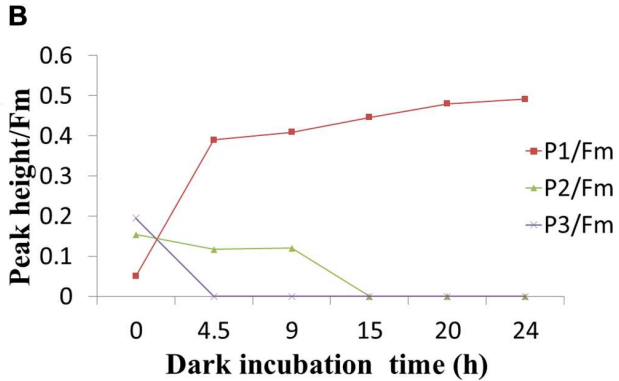

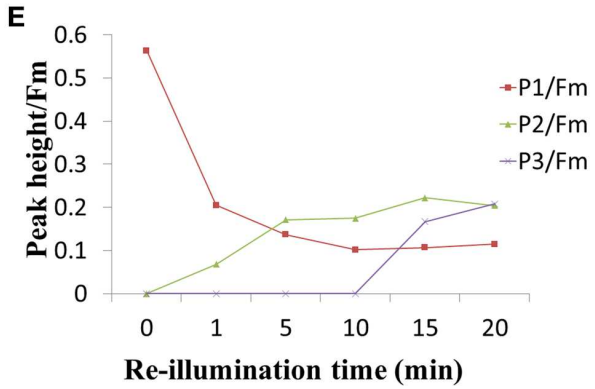

FIGURE 2 | Effect of dark starvation and re-illumination on the oscillation kinetics of post-illumination increase in Chl fluorescence in WT Synechocystis PCC 6803. After the cell at mid-logarithmic stage (OD730 $\approx 0.5$ ) was incubated in the dark for indicated times, the kinetics was measured. (A) Dark-starvation for 9 h; (C) dark-starvation for 20 h; (B) the time course of changes in peak1 (P1), peak2 (P2), and peak3 (P3) during dark adaption; (D) the kinetics of the 20 h-dark-starved cells after re-illumination for 20 min; (E) the time course of the changes in P1, P2, and P3 after re-illumination at different times. The measurement conditions were the same as in Figure 1. Every experiment was independently repeated three times at least.

at $34^{\circ} \mathrm{C}$. As shown in Figure 3B, the growth of M55 was much slower as compared to the wild type and could not further grow after 10 days. The result suggests that the enhancement of both fast and slow phases related with the cyclic electron flow around PS I mediated by NDH-1 is required for the heat tolerance for the Synechocystis PCC 6803.

\section{Effect of $\mathrm{NaCl}$ Stress on the Oscillation Kinetics of Post-illumination Increase in Chl Fluorescence}

It has been demonstrated that NDH-1 dependent cyclic electron flow is essential for short-term adaptation of Synechocystis to salt shock (Tanaka et al., 1997). The response of the oscillation kinetics to salt stress was investigated. As shown in Figure 3C, the P2 was significantly increased, reached to the maximum at $1 \mathrm{~h}$ and then gradually decreased. P1 was only slightly stimulated, but P3 was decreased after incubation of the cells in $0.8 \mathrm{M}$ $\mathrm{NaCl}$. By comparison with the wild type, M55 could not grow in the culture medium contained $0.8 \mathrm{M} \mathrm{NaCl}$ (Figure 3D), suggesting that the stimulated $\mathrm{P} 2$ related with $\mathrm{NDH}$-pathway is essential for salt stress response and tolerance in Synechocystis PCC 6803.

\section{DISCUSSION}

Post-illumination increase in Chl fluorescence is widely used for investigation of the activity of cyclic electron flow around
PS I mediated by NDH-1, which reflects the reduction of PQ pool by the electrons from the photoreductants generated in PS I during illumination in cyanobacteria (Mi et al., 1995; Deng et al., 2003) and high plants (Sazanov et al., 1998; Shikanai et al., 1998). This work studied the oscillation kinetics of the post-illumination increase in Chl fluorescence for first time. The significant enhancement of the fast phase by adding the cofactor (PMS) of cyclic electron flow (Figure 1) or by dark incubation (Figure 2), indicating that the fast phase of the kinetics reflects the donation of electrons to PQ pool from the photoreductants generated in PS I, such as reduced fd, NADPH, that is the short-cut cyclic electron flow around PS I. Moreover, the increase of the fast phase of kinetics but suppression of slow phase in presence of high concentration of IA (Figure 1), an inhibitor of Calvin cycle, as well as the re-appearance of the slow phases after re-illumination the starved cells for $20 \mathrm{~min}$ (Figure 2) indicate that the slow phase may reflect the donation of electrons from the photoreductants generated in process of carbon assimilation, probably triose phosphates, glucose. It has been proposed the existence of a large pool of electron donors when illuminating the dark starvation cells of Synechocystis PCC 6803 by AL continues for more than $10 \mathrm{~s}$, as analyzed by the complementary areas of the re-oxidation curve of P700 by far-red light after illumination with AL (Mi et al., 1994). Thus, the slow phase kinetics of the post-illumination increase in $\mathrm{Chl}$ fluorescence probably represents the reduction of the PQ pool by such a large pool of electrons accumulated in stroma or cytosol 

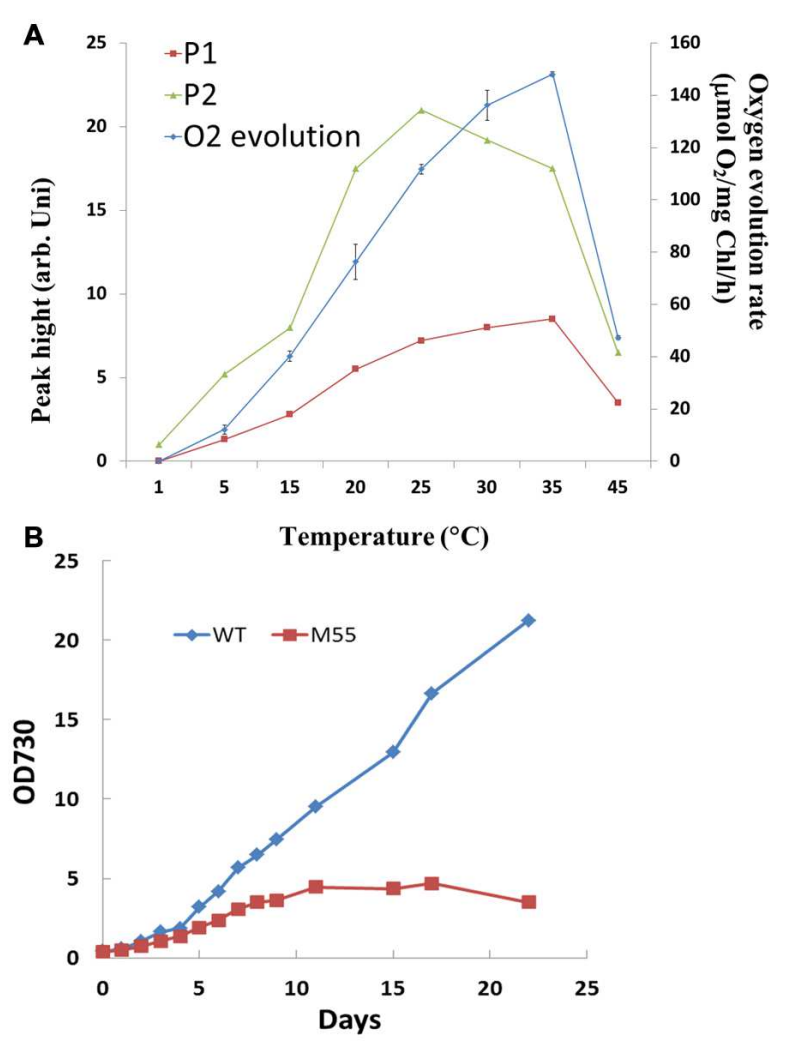
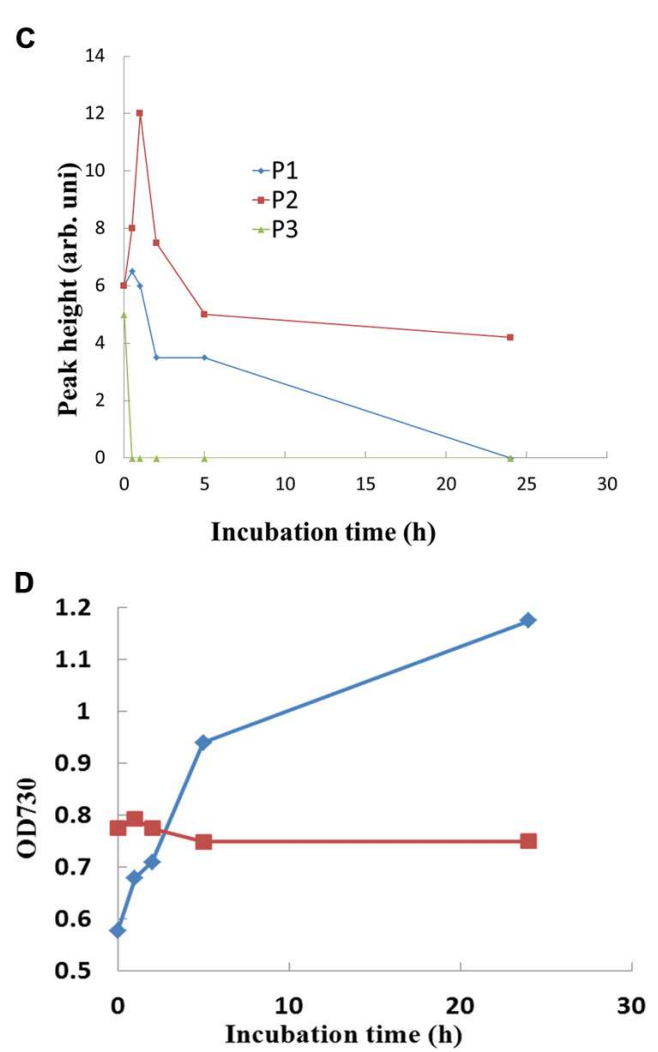

FIGURE 3 | Effect of temperature (A) and high concentration of $\mathrm{NaCl}(\mathrm{C})$ on the oscillation kinetics of post-illumination increase in Chl fluorescence in WT Synechocystis PCC 6803. Comparison of the effect of high temperature $\left(34^{\circ} \mathrm{C}\right)$ (B) and $0.8 \mathrm{M} \mathrm{NaCl}$ (D) on the growth between WT and M55. The measurement conditions were the same as in Figure 1. Every experiment was independently repeated three times at least.

during illumination. Both the fast phase and slow phases were not detectable in NdhB defective mutant, M55, even in the presence of cyclic co-factor PMS (Figure 1), suggesting that $\mathrm{NDH}-1$ is involved in the mediation of electron donation to the PQ pool from different substrates. The previous study has shown that the activity of both the respiratory flow and cyclic electron flow is light-dependent (Mi et al., 2001), therefore, the light inducible slow phases might be reflected the redox states of PQ pool by the electron donation from the respiratory flow as well as the cyclic electron flow.

As one of photoprotective pathways, cyclic electron flow around PS I mediated by $\mathrm{NDH}$ has been found to be enhanced under stressed conditions, such as high temperature (Wang et al., 2006), strong light (Mi et al., 2001), salt shock (Tanaka et al., 1997). In our results, the increase of both the fast and slow phases as well as the enhanced activity of photosynthetic oxygen evolution at higher physiological temperature (Figure 3A) suggests that the cyclic electron flow has close relationship with activity of $\mathrm{CO}_{2}$ assimilation under stressed condition. It has been indicated that cyclic electron flow around PS I plays an important role in providing ATP for carbon assimilation (Schurman et al., 1972; Slovacek and Hind, 1981). Actually, the ATP supply could not always satisfy the demand of the plant during the later grain filling stage (Shen, 1994). Evidence to support this suggestion is that the enhancement of cyclic electron flow around PS I by treatment with low concentration of $\mathrm{NaHSO}_{3}$ enables plants or cyanobacteria to generate sufficient proton gradient across the thylakoid membrane and ATP (Wu et al., 2011; Rolfe et al., 2012), thereby increased the biomass of cyanobacterium Synechocystis PCC 6803 (Wang et al., 2003). The research of mutants impaired in both NDH- and PGR5-pathways have indicated that cyclic electron flow around PS I is required for the efficient photosynthesis (Munekage et al., 2002). Thus, the growth of M55 was severely suppressed at $34^{\circ} \mathrm{C}$ when the activity of cyclic electron flow around PS I mediated by NDH-1 is impaired by knocking out the $N d h B$ gene (Figure 3B). Salinity stress is one of the most serious factors limiting the productivity of plants. One of strategies to adapt salinity condition is to exclude sodium ion from cytosol by $\mathrm{Na}^{+} / \mathrm{H}^{+}$antiporter $\mathrm{Na}^{+}$ATPase in plants (Blumwald and Poole, 1987; Apse et al., 1999) or in cyanobacteria (Kaku et al., 1999). The exclusion of $\mathrm{Na}^{+}$ is an energy consuming process, while cyclic electron flow around PS I provides extra ATP in this demand. Based on the stimulation of the donation of electrons from the cytosol to $\mathrm{P} 700^{+}$via $\mathrm{NDH}-1$, it has been suggested that the cyclic 
electron flow mediated by NDH-1 might play an important role in the exclusion of $\mathrm{Na}^{+}$ions from salt-shocked cells of Synechocystis PCC 6803 (Tanaka et al., 1997). The significantly stimulated slow phase, P2, at high salt stress (Figure 3C) indicates the large electron pool contributed to the cyclic electron flow may function for providing ATP for salinity tolerance. Impairment of both the fast and slow phases in M55 increased the sensitivity to the high salt stressed conditions (Figure 3D), supporting the above mention.

\section{CONCLUSION}

The studying the oscillation kinetics of the postillumination increase in Chl fluorescence enables us to distinguish the different phases as the indicators for electron donations to the PQ pool from different photoreductants via NDH-mediated cyclic electron flow around PS I, and to use the kinetics to analyze the physiological responses to environmental stresses in cyanobacteria.

\section{REFERENCES}

Allen, M. M. (1968). Simple conditions for growth of unicellular blue-green algae on plates. J. Phycol. 4, 1-4. doi: 10.1111/j.1529-8817.1968.tb04667.x

Apse, M. P., Aharon, G. S., Snedden, W. A., and Blumwald, E. (1999). Salt tolerance conferred by overexpression of a vacuolar $\mathrm{Na}^{+} / \mathrm{H}^{+}$antiport in Arabidopsis. Science 285, 1256-1258. doi: 10.1126/science.285.5431.1256

Asada, K., Heber, U., and Schreiber, U. (1993). Electron flow to the intersystem chain from stromal components and cyclic electron flow in maize chloroplasts, as detected in intact leaves by monitoring redox change of P700 and chlorophyll fluorescence. Plant Cell Physiol. 34, 39-50.

Battchikova, N., Wei, L., Du, L., Bersanini, L., Aro, E.-M., and Ma, W. (2012). Identification of novel Ssl0352 protein (NdhS), essential for efficient operation of cyclic electron transport around photosystem I, in NADPH: plastoquinone oxidoreductase (NDH-1) complexes of Synechocystis sp. PCC 6803. (vol 286, pg 36992, 2011). J. Biol. Chem. 287, 8660-8660. doi: 10.1074/jbc.M111.263780

Battchikova, N., Zhang, P. P., Rudd, S., Ogawa, T., and Aro, E. M. (2005). Identification of NdhL and Ssl1690 (NdhO) in NDH-1L, and NDH-1M complexes of Synechocystis sp PCC 6803. J. Biol. Chem. 280, 2587-2595. doi: 10.1074/jbc.M410914200

Birungi, M., Folea, M., Battchikova, N., Xu, M., Mi, H., Ogawa, T., et al. (2010). Possibilities of subunit localization with fluorescent protein tags and electron microscopy examplified by a cyanobacterial NDH-1 study. Biochim. Biophys. Acta 1797, 1681-1686. doi: 10.1016/j.bbabio.2010.06.004

Blumwald, E., and Poole, R. J. (1987). Salt tolerance in suspension-cultures of sugar-beet - Induction of $\mathrm{NA}^{+} / \mathrm{H}^{+}$antiport activity at the tonoplast by growth in salt. Plant Physiol. 83, 884-887. doi: 10.1104/pp.83.4.884

Deng, Y., Ye, J. Y., and Mi, H. L. (2003). Effects of low CO2 on NAD(P)H dehydrogenase, a mediator of cyclic electron transport around photosystem I in the cyanobacterium Synechocystis PCC6803. Plant Cell Physiol. 44, 534-540. doi: $10.1093 / \mathrm{pcp} / \mathrm{pcg} 067$

Friedrich, T., and Scheide, D. (2000). The respiratory complex I of bacteria, archaea and eukarya and its module common with membrane-bound multisubunit hydrogenases. FEBS Lett. 479, 1-5. doi: 10.1016/S0014-5793(00)01867-6

Hatch, M. D. (1987). C-4 photosynthesis: a unique blend of modified biochemistry, anatomy and ultrastructure. Biochim. Biophys. Acta 895, 81-106. doi: 10.1016/S0304-4173(87)80009-5

Joliot, P., and Alric, J. (2013). Inhibition of CO2 fixation by iodoacetamide stimulates cyclic electron flow and non-photochemical quenching upon far-red illumination. Photosyn. Res. 115, 55-63. doi: 10.1007/s11120-013-9826-1

\section{AUTHOR CONTRIBUTIONS}

All authors listed, have made substantial, direct and intellectual contribution to the work, and approved it for publication. MX performed the research work and manuscript revision. JL helped performing experiments. PF discussed for the study. HM designed the research and wrote the paper.

\section{FUNDING}

This work was supported by grants from the State Key Basic Research and Development Plan 973 (No. 2013CB127005, 2015CB150104, and 2011C13200902), the National Natural Scientific Foundation of China (31470338) and Shanghai Science Foundation [13DJ1400102].

\section{ACKNOWLEDGMENT}

We thank retired Prof. Ogawa T in Nagoya University, Japan for providing M55 strain.

Jones, L. W., and Myers, J. (1963). A common link between photosynthesis and respiration in a blue-green alga. Nature 199, 670-672. doi: 10.1038/199 $670 \mathrm{a} 0$

Kaku, N., Hibino, T., Tanaka, Y., Takabe, T., Nakamura, T., and Takabe, T. (1999). Expression of nhaAv gene encoding $\mathrm{Na}^{+} / \mathrm{H}^{+}$antiporter from Vibrio alginolyticus in a freshwater cyanobacterium Synechococcus sp PCC 7942 confers lithium tolerance, but not sodium tolerance. Plant Cell Physiol. 40, 557-564. doi: 10.1093/oxfordjournals.pcp.a029577

Mano, J., Miyake, C., Schreiber, U., and Asada, K. (1995). Photoactivation of the electron flow from NADPH to plastoquinone in spinach chloroplasts. Plant Cell Physiol. 36, 1589-1598.

Mi, H., Endo, T., Schreiber, U., Ogawa, T., and Asada, K. (1994). NAD(P)H Dehydrogenase-dependent cyclic electron flow around Photosystem-I in the cyanobacterium Synechocystis PCC-6803: a study of dark-starved cells and spheroplasts. Plant Cell Physiol. 35, 163-173.

Mi, H. L., Deng, Y., Tanaka, Y., Hibino, T., and Takabe, T. (2001). Photoinduction of an NADPH dehydrogenase which functions as a mediator of electron transport to the intersystem chain in the cyanobacterium Synechocystis PCC6803. Photosyn. Res. 70, 167-173. doi: 10.1023/A:1017946524199

Mi, H. L., Endo, T., Ogawa, T., and Asada, K. (1995). Thylakoid membrane-bound, NADPH-specific pyridine-nucleotide dehydrogenase complex mediates cyclic electron-transport in the cyanobacterium Synechocystis sp PCC-68038. Plant Cell Physiol. 36, 661-668.

Mi, H. L., Endo, T., Schreiber, U., Ogawa, T., and Asada, K. (1992). Electron donation from cyclic and respiratory flows to the photosynthetic intersystem chain is mediated by pyridine-nucleotide dehydrogenase in the cyanobacterium Synechocystis PCC-6803. Plant Cell Physiol. 33, 1233-1237.

Mills, J. D., Slovacek, R. E., and Hind, G. (1978). Cyclic electron-transport in isolated intact chloroplasts - Further-studies with antimycin. Biochim. Biophys. Acta 504, 298-309. doi: 10.1016/0005-2728(78)90178-0

Munekage, Y., Hojo, M., Endo, T., and Shikanai, T. (2002). Arabidopsis pgr5 is defective in cyclic electron flow around photosystem I. Plant Cell Physiol. 43, S23-S23.

Nowaczyk, M. M., Wulfhorst, H., Ryan, C. M., Souda, P., Zhang, H., Cramer, W. A., et al. (2011). NdhP and NdhQ: two novel small subunits of the cyanobacterial NDH-1 complex. Biochemistry 50, 1121-1124. doi: 10.1021/bi102044b

Ogawa, T. (1991). A gene homologous to the subunit-2 gene of $\mathrm{NADH}$ dehydrogenase is essential to inorganic carbon transport of Synechocystis PCC6803. Proc. Natl. Acad. Sci. U.S.A. 88, 4275-4279. doi: $10.1073 /$ pnas.88.10.4275 
Prommeenate, P., Lennon, A. M., Markert, C., Hippler, M., and Nixon, P. J. (2004). Subunit composition of NDH-1 complexes of Synechocystis sp PCC 6803 - Identification of two new ndh gene products with nuclear-encoded homologues in the chloroplast Ndh complex. J. Biol. Chem. 279, 28165-28173. doi: 10.1074/jbc.M401107200

Rolfe, M. D., Ocone, A., Stapleton, M. R., Hall, S., Trotter, E. W., Poole, R. K., et al. (2012). Systems analysis of transcription factor activities in environments with stable and dynamic oxygen concentrations. Open Biol. 2, 120091. doi: 10.1098/rsob.120091

Sazanov, L. A., Burrows, P. A., and Nixon, P. J. (1998). The plastid ndh genes code for an NADH-specific dehydrogenase: isolation of a complex I analogue from pea thylakoid membranes. Proc. Natl. Acad. Sci. U.S.A. 95, 1319-1324. doi: 10.1073/pnas.95.3.1319

Scherer, S. (1990). do photosynthetic and respiratory electron-transport chains share redox proteins. Trends Biochem. Sci. 15, 458-462. doi: 10.1016/09680004(90)90296-N

Scherer, S., Almon, H., and Boger, P. (1988). Interaction of photosynthesis, respiration and nitrogen-fixation in cyanobacteria. Photosyn. Res. 15, 95-114. doi: 10.1007/BF00035255

Schreiber, U., Bilger, W., and Neubauer, C. (1994). "Chlorophyll fluorescence as a non-intrusive indicator for rapid assessment of in vivo photosynthesis," in Ecophysiology of Photosynthesis, eds E.-D. Schulze and M. M. Caldwell (Berlin: Springer-Verlag), 49-70.

Schreiber, U., Hormann, H., Neubauer, C., and Klughammer, C. (1995). Assessment of photosystem II photochemical quantum yield by chlorophyll fluorescence quenching analysis. Aust. J. Plant Physiol. 22, 209-220. doi: 10.1071/PP9950209

Schurman, P., Buchanan, B. B., and Arnon, D. I. (1972). Role of cyclic photophosphorylation in photosynthetic carbon-dioxide assimilation by isolated chloroplasts. Biochim. Biophys. Acta 267, 111-124. doi: 10.1016/00052728(72)90143-0

Schwarz, D., Schubert, H., Georg, J., Hess, W. R., and Hagemann, M. (2013). The gene sml0013 of Synechocystis species strain PCC 6803 encodes for a novel subunit of the $\mathrm{NAD}(\mathrm{P}) \mathrm{H}$ oxidoreductase or complex $\mathrm{i}$ that is ubiquitously distributed among Cyanobacteria. Plant Physiol. 163, 1191-1202. doi: $10.1104 /$ pp.113.224287

Shen, Y. K. (1994). Dynamic approaches to the mechanism of photosynthesis. Photosynth. Res. 39, 3-13. doi: 10.1007/BF00027138

Shikanai, T., Endo, T., Hashimoto, T., Yamada, Y., Asada, K., and Yokota, A. (1998). Directed disruption of the tobacco ndhB gene impairs cyclic electron flow around photosystem I. Proc. Natl. Acad. Sci. U.S.A. 95, 9705-9709. doi: 10.1073/pnas.95.16.9705

Slovacek, R. E., and Hind, G. (1981). Correlation between photosynthesis and the transthylakoid proton gradient. Biochim. Biophys. Acta 635, 393-404. doi: 10.1016/0005-2728(81)90037-2

Slovacek, R. E., Mills, J. D., and Hind, G. (1978). Function of cyclic electrontransport in photosynthesis. FEBS Lett. 87, 73-76. doi: 10.1016/00145793(78)80136-7

Tanaka, Y., Katada, S., Ishikawa, H., Ogawa, T., and Takabe, T. (1997). Electron flow front $\mathrm{NAD}(\mathrm{P}) \mathrm{H}$ dehydrogenase to photosystem I is required for adaptation to salt shock in the Cyanobacterium Synechocystis sp. PCC 6803. Plant Cell Physiol. 38, 1311-1318.

Trebst, A. (1974). Energy conservation in photosynthetic electron-transport of chloroplasts. Annu. Rev. Plant Physiol. Plant Mol. Biol. 25, 423-458. doi: 10.1146/annurev.pp.25.060174.002231

Wang, H.-W., Mi, H., Ye, J.-Y., Deng, Y., and Shen, Y.-K. (2003). Low concentrations of NaHSO3 increase cyclic photophosphorylation and photosynthesis in cyanobacterium Synechocystis PCC6803. Photosynth. Res. 75, 151-159. doi: 10.1023/A:1022813402265

Wang, P., Duan, W., Takabayashi, A., Endo, T., Shikanai, T., Ye, J. Y., et al. (2006). Chloroplastic NAD(P)H dehydrogenase in tobacco leaves functions in alleviation of oxidative damage caused by temperature stress. Plant Physiol. 141, 465-474. doi: 10.1104/pp.105.070490

Wu, Y., Zheng, F., Ma, W., Han, Z., Gu, Q., Shen, Y., et al. (2011). Regulation of NAD(P)H dehydrogenase-dependent cyclic electron transport around PSI by $\mathrm{NaHSO}(3)$ at low concentrations in tobacco chloroplasts. Plant Cell Physiol. 52, 1734-1743. doi: 10.1093/pcp/pcr109

$\mathrm{Xu}, \mathrm{M}$., Shi, N., Li, Q., and Mi, H. (2014). An active supercomplex of NADPH dehydrogenase mediated cyclic electron flow around Photosystem I from the panicle chloroplast of Oryza sativa. Acta Biochim. Biophys. Sin. 46, 757-765. doi: 10.1093/abbs/gmu064

Yamamoto, H., Peng, L., Fukao, Y., and Shikanai, T. (2011). An Src homology 3 domain-like fold protein forms a ferredoxin binding site for the chloroplast NADH dehydrogenase-like complex in Arabidopsis. Plant Cell 23, 1480-1493. doi: $10.1105 /$ tpc. 110.080291

Yamamoto, H., and Shikanai, T. (2013). In planta mutagenesis of Src homology 3 domain-like fold of NdhS, a ferredoxin-binding subunit of the chloroplast NADH dehydrogenase-like complex in Arabidopsis: a conserved Arg-193 plays a critical role in ferredoxin binding. J. Biol. Chem. 288, 36328-36337. doi: 10.1074/jbc.M113.511584

Zhang, J., Gao, F., Zhao, J., Ogawa, T., Wang, Q., and Ma, W. (2014). NdhP is an exclusive subunit of large complex of NADPH dehydrogenase essential to stabilize the complex in Synechocystis sp strain PCC 6803. J. Biol. Chem. 289, 18770-18781. doi: 10.1074/jbc.M114.55 3404

Zhao, J., Rong, W., Gao, F., Ogawa, T., and Ma, W. (2015). Subunit Q is required to stabilize the large complex of NADPH dehydrogenase in Synechocystis sp strain PCC 6803. Plant Physiol. 168, 443-451. doi: 10.1104/pp.15. 00503

Conflict of Interest Statement: The authors declare that the research was conducted in the absence of any commercial or financial relationships that could be construed as a potential conflict of interest.

Copyright (C) $2016 \mathrm{Xu}, \mathrm{Lv}, \mathrm{Fu}$ and Mi. This is an open-access article distributed under the terms of the Creative Commons Attribution License (CC BY). The use, distribution or reproduction in other forums is permitted, provided the original author(s) or licensor are credited and that the original publication in this journal is cited, in accordance with accepted academic practice. No use, distribution or reproduction is permitted which does not comply with these terms. 